\title{
¿Interpretan los alumnos de ingeniería química los gráficos logarítmicos correctamente? Desarrollo de una herramienta para mejorar su precisión
}

\author{
Manuel César Martí-Calatayud ${ }^{\mathrm{a}}$, Asunción Santafé-Moros ${ }^{\mathrm{a}}$ y José M. Gozálvez-Zafrilla ${ }^{\mathrm{a}}$ \\ ${ }^{a}$ Departamento de Ingeniería Química y Nuclear. Instituto de Seguridad Industrial, Radiofísica y Medioambiental. \\ E.T.S.E.I. de la Universitat Politècnica de València,jmgz@iqn.upv.es, assanmo@iqn.upv.es, mcmarti@iqn.upv.es
}

\begin{abstract}
The interpretation of logarithmic plots is an important skill for engineers. Such graphical representations are widely used to show the evolution of natural phenomena and to facilitate the design of processes and equipments. The fact that engineering students are more familiar with the interpretation of linear-scale diagrams implies a challenge during the learning process of reading other types of representations. In the present work, the interpretation skills of logarithmic plots by chemical engineering undergraduates is evaluated. The analysis over a sample of 64 students reveals that 49\% of them make reading mistakes, being the interpretation of linear scales and the incorrect count of minor grid lines the most common ones. With the aim of assisting the students with the use of logarithmic-scale plots, we have developed a software capable of reading values from different engineering plots; which we called PUNTGRAF. The program can be used to calibrate different types of charts and to obtain point coordinates. We propose PUNTGRAF as a useful tool to reinforce the interpretation skills of engineering plots by undergraduate students, which would help them to learn from their own mistakes in a self-training process.
\end{abstract}

Keywords: logarithmic plots, graph interpretation, puntgraf, chart reading, chemical engineering, graph comprehension, science education

\section{Resumen}

La interpretación de gráficos logaritmicos es una habilidad importante en los profesionales de ingeniería. Dichos gráficos se utilizan para representar la evolución de fenómenos naturales y para facilitar el diseño de procesos y equipos. No obstante, los estudiantes están acostumbrados a leer datos en diagramas con escalas lineales, lo cual supone un reto en el aprendizaje de la lectura de otros tipos de representaciones. En el presente trabajo se evalúan las capacidades de interpretación de gráficos logaritmicos en estudiantes de tercer curso de grado de ingeniería química. El análisis sobre una muestra de 64 individuos revela que un 49\% de los alumnos comete errores de interpretación, siendo los mas comunes la lectura de datos realizando una interpretación de escalas lineal y el conteo erróneo del número de líneas de división secundarias. Con el fin de facilitar el manejo de diagramas con escalas logarítmicas, se ha desarrollado un software de lectura de gráficos llamado PUNTGRAF. El programa permite calibrar distintos tipos de gráficos, y obtener las coordenadas de un punto. Se propone PUNTGRAF como una herramienta de apoyo en el aprendizaje de la interpretación de gráficos ingenieriles, que permita al alumno aprender de forma autónoma de sus propios errores.

Palabras clave: gráficos logarítmicos, interpretación de gráficos, puntgraf, lectura de gráficos, ingeniería química 


\section{Introducción}

La interpretación y obtención de datos a partir de gráficos es una habilidad necesaria en multitud de problemas de ingeniería, tanto para el diseño de equipos, como en la realización de cálculos físicos o en el análisis de procesos. En los estudios universitarios de ingeniería química, en los cuales se enmarca el presente trabajo, la obtención de datos a partir de diferentes tipos de diagramas es una competencia multidisciplinar, dado que se aplica en materias tan variadas como las relacionadas con reactores químicos, operaciones de separación, o termodinámica química aplicada.

N. Glazer incluyó la obtención e interpretación de datos a partir de representaciones gráficas entre las competencias que los estudiantes de ingeniería necesitan aplicar no solamente en el entorno del aula, sino de forma continuada a lo largo de su trayectoria profesional (Glazer, 2011). Asimismo, también remarcó las dificultades que suelen encontrar los estudiantes en dichas tareas debido a la variedad en la tipología de representaciones gráficas a las que se pueden enfrentar. A este respecto, Dreyfus y Eisenberg indican que la comprensión de gráficos es una tarea compleja que no ocurre de forma automática, sino que debe ser aprendida; no pudiendo este hecho ser obviado por los docentes universitarios (Dreyfus \& Eisenberg, 1990).

Cabe tener en cuenta que un aprendizaje deficiente de la interpretación de gráficos en profesionales de ingeniería química puede implicar errores graves en el diseño y operación de equipos e instalaciones. Además, en múltiples situaciones la tarea de lectura no consiste en la simple obtención de un valor discreto a partir de un gráfico; sino que puede requerir, por ejemplo, la medida del segmento que une dos puntos para aplicar la regla de la palanca, o el cálculo de pendientes. En tales casos, la imprecisión en la lectura de varios datos puede magnificar el error cometido al resolver un problema de ingeniería.

Normalmente, el alumnado accede a los estudios universitarios sin haber trabajado la interpretación y obtención de datos a partir de gráficos de distinta tipología. La experiencia previa referente a la lectura de diagramas se centra fundamentalmente en gráficos con forma rectangular, en los cuales se representa una variable dependiente (y) en el eje de ordenadas en función de otra variable (x) utilizando escalas lineales. No obstante, en ingeniería existen otros tipos de representaciones gráficas de uso frecuente. Tal es el caso de los gráficos triangulares, en los cuales se representan las composiciones de sistemas ternarios; o el de los diagramas logarítmicos y semilogarítmicos, en los cuales, incrementos proporcionales de una o dos de las escalas se representan por factores usualmente de 10. En concreto, los gráficos logarítmicos son muy recurrentes cuando es necesario mostrar la evolución de una variable en un rango muy amplio de valores positivos. Asimismo, estos gráficos también conllevan un nivel de dificultad mayor para su interpretación. A continuación se enumeran algunos ejemplos de los diagramas logarítmicos más utilizados en el campo de la ingeniería química:

- Gráficos de equilibrios químicos en función del pH $\left(-\log \left[\mathrm{H}^{+}\right]\right)$

- Correlaciones de coeficientes de transferencia de masa

- Gráficos termodinámicos donde se representa la presión de vapor de compuestos químicos frente a la temperatura

- Diagramas de Moody en los que se representa la evolución de un factor de fricción en función del del número adimensional Reynolds

- Representaciones de la eficiencia de etapas para absorbedores de gases en función de números adimensionales

- Diagramas cinéticos en los que se representa la eficiencia de distintos catalizadores en función del módulo de Thiele 
Pese a que existe una predisposición natural en los humanos a interpretar cambios de variables en escalas lineales, multitud de procesos naturales también tienen lugar en forma de caídas o incrementos exponenciales. En el campo de las ciencias naturales, existen diversas propuestas docentes basadas en laboratorios de química o de física para inculcar la comprensión de dichos procesos y familiarizar a los alumnos con el uso de representaciones logarítmicas (DePierro, Garafalo, \& Toomey, 2008; Di Capua, Offi, \& Fontana, 2014; Park \& Choi, 2013). En los estudios de Ingeniería Química, la lectura de diagramas con escala logarítmica se suele tratar de forma accesoria en la resolución de algunos problemas muy específicos de diferentes asignaturas. Este hecho puede implicar que, si no se hace suficiente hincapié en la asimilación del concepto de escala logarítmica, los ingenieros químicos realicen interpretaciones erróneas, incluso durante su desempeño como profesionales. En este sentido, Heckler y colaboradores investigaron los tipos de error más comunes en la lectura de diagramas logarítmicos cometidos por estudiantes participantes en un curso preuniversitario de ciencia de materiales en la Universidad estatal de Ohio, observando que solamente un 30-40\% de los alumnos realizaban una lectura precisa de los gráficos (Heckler, Mikula, \& Rosenblatt, 2013). En dicho estudio, se comprobó que tras la realización de tareas online distribuidas a lo largo del curso, la precisión de los alumnos mejoró hasta el $80 \%$. Por tanto, el desarrollo de herramientas que permitan al alumno identificar sus errores en la lectura de diagramas logarítmicos puede suponer un recurso transversal utilizable en distintas asignaturas y que se traduzca en curvas de aprendizaje más rápidas y eficientes.

En el presente trabajo se han evaluado los errores más comunes en la lectura de gráficos logarítmicos cometidos por alumnos universitarios de Ingeniería Química. Además, se presenta una herramienta informática desarrollada para que el alumno pueda percatarse de forma autónoma de los errores cometidos y mejore su precisión en la lectura de gráficos de distinto tipo.

\section{Objetivos}

Los tres objetivos principales de este trabajo son:

- Determinar los errores más comunes cometidos por alumnos del grado de ingeniería química en la lectura de diagramas logarítmicos

- Desarrollar una aplicación informática que permita a los alumnos obtener datos precisos a partir de gráficos de distinta tipología, entre ellos los diagramas logarítmicos

- Poner a disposición de los alumnos dicha aplicación para la comprobación de los errores cometidos y el perfeccionamiento de la interpretación de distintos tipos de gráficos.

\section{Desarrollo de la innovación}

\subsection{Contexto de aplicación de la presente investigación}

La presente investigación docente se ha llevado a cabo con alumnos de tercer curso del grado en Ingeniería Química de la Universitat Politècnica de València. En concreto, se ha trabajado con alumnos que cursaban la asignatura Experimentación en Ingeniería Química 2. La asignatura tiene un carácter marcadamente experimental, en la cual, los alumnos realizan ensayos en el laboratorio relacionados con el contenido teórico de otras asignaturas. A posteriori, deben tratar los datos experimentales recabados y presentarlos en un informe. Los contenidos tratados son variados, abarcando conceptos relacionados con las operaciones de separación o la cinética química. En dicha etapa curricular, a los alumnos se les 
presupone el conocimiento y la capacidad de interpretación de diagramas logarítmicos, dada su utilización en asignaturas de cursos anteriores, tales como Estadística o Termodinámica.

En una de las prácticas de la asignatura, los alumnos deben encontrar las condiciones de un reactor de mezclado que optimizan el grado de homogeneidad de una suspensión de carbonato cálcico. Para ello, se analizan distintas muestras recogidas a distintas alturas de un reactor, el cual se encuentra agitado con un rodete. Se prueban distintas velocidades de giro, con y en ausencia de placas deflectoras, cuya función es la supresión de vórtices. Durante la sesión de tratamiento de datos, los alumnos se enfrentan a un problema típico de ingeniería, en el cual deben calcular la potencia consumida por el sistema de agitación mediante el cálculo del número adimensional de Reynolds. Con el número de Reynolds, los alumnos entran al eje de abcisas de un diagrama logarítmico, donde cortando con la curva correspondiente al rodete utilizado, pueden leer el factor de potencia en el eje de las ordenadas. Los alumnos trabajan en equipos de 3 a 4 personas, pudiendo consultar las dudas con el profesor responsable durante la sesión de tratamiento de datos. Al cabo de una semana, cada grupo recibe sus memorias corregidas con indicaciones de los errores cometidos, con el fin de que no los repitan en el examen de la asignatura.

\subsection{Interpretación de gráficos logarítmicos}

Los errores cometidos por los alumnos durante la utilización de diagramas logarítmicos se evaluaron a través de una de las preguntas realizadas en el examen, cuyo procedimiento de resolución es similar al seguido en la práctica de agitación. Los alumnos debían calcular el número de Reynolds correspondiente a una suspensión sujeta a unas condiciones de agitación y obtener, mediante la ayuda de un díagrama (Figura 1), la potencia consumida durante el proceso. El diagrama, que es análogo al diagrama de Moody para estimación del factor de fricción, relaciona el número adimensional de potencia de agitación con un número de Reynolds para condiciones de agitación. El grafico proporcionado en el examen fue el de la figura mostrada. Se puede observar que cuenta con una sóla curva correspondiente al tipo de agitador utilizado, representándose ambos ejes en escala logarítmica. Además, el gráfico incluye líneas de lectura auxiliares horizontales y verticales. La muestra sobre la cual se realizó la presente investigación está constituida por los 64 alumnos que se presentaron al examen.

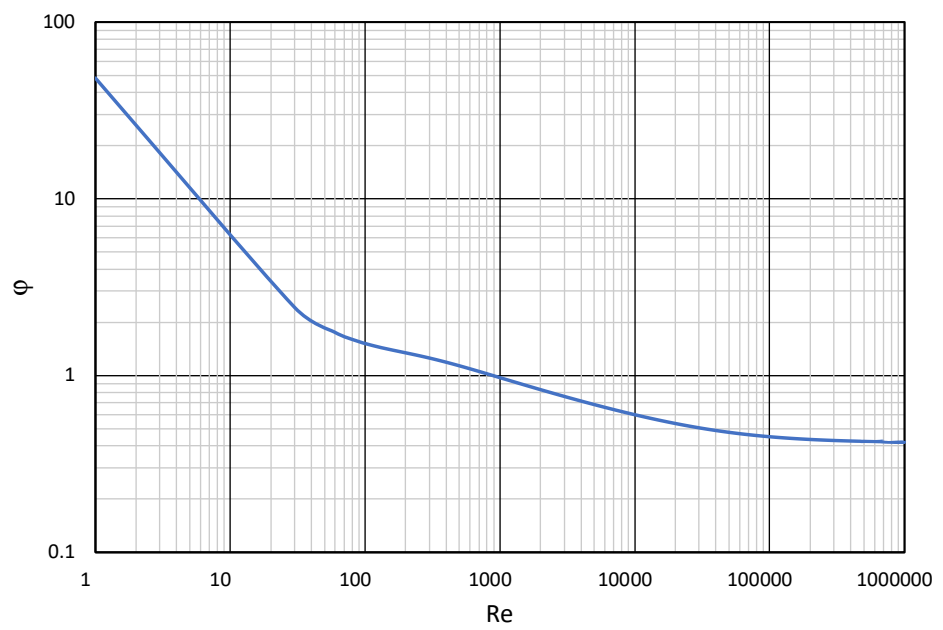

Fig. 1 Diagrama logaritmico en el cual se representa el número de potencia, $\varphi$, frente al número de Reynolds, Re, para un agitador de hélice. Fuente de la correlación: (Furukawa et al., 2012). 


\subsection{Desarrollo del software y su utilización}

Con el fin de proporcionar una herramienta para realizar lecturas en distintos tipos de gráficos, se desarrolló una aplicación ejecutable en Matlab llamada PUNTGRAF. La aplicación permite cargar archivos en formato imagen. Los alumnos pueden utilizar dicha aplicación para realizar lecturas precisas de distintos gráficos ingenieriles. Otra posible utilidad, de carácter docente, es su utilización a modo de comparación con las lecturas realizadas por los alumnos. De este modo, éstos serán capaces de identificar qué conceptos no tienen claros, en qué se equivocan, y podrán aprender el procedimiento correcto para interpretar los gráficos logarítmicos adecuadamente.

\section{Resultados}

\subsection{Interpretación de gráficos logarítmicos}

En el ejercicio de examen los alumnos necesitaban calcular el número de Reynolds de un sistema de agitación. Una vez calculado dicho número, debían entrar en la gráfica de la Figura 1 y cortar con la curva del rodete (representada en azul), leyendo finalmente en el eje y el valor del factor de potencia. Los datos necesarios para el cálculo del número de Reynolds, tales como la velocidad de giro del rodete o la densidad de la disolución, se adaptaron para que el valor de entrada al gráfico cumpliera las siguientes condiciones:

- El valor es un múltiplo entero de una de las líneas de división principales, de forma que los alumnos pueden entrar al gráfico guiándose con una línea de división secundaria.

- El valor debe estar alejado de las líneas principales de división, con el fin de poder evaluar fácilmente si la interpretación de la escala logarítmica es correcta.

Siendo el valor calculado del número $\mathrm{Re}=40000$, en la Figura 2 se muestra un zoom del gráfico logarítmico proporcionado, donde la flecha indica la solución correcta (valor de $\varphi \approx 0.49$ ). Tras la corrección del ejercicio de examen se procedió a identificar los errores más comunes y a clasificar las respuestas de los alumnos en cuatro grandes grupos:

- Alumnos que resuelven el problema correctamente, sabiendo por tanto, extraer el valor adecuado del diagrama logarítmico.

- Alumnos que no realizan correctamente el cálculo del número de Reynolds. Estos alumnos se excluyen del posterior análisis, puesto que no es posible evaluar sus capacidades de interpretación de diagramas logarítmicos.

- Alumnos que realizan una interpretación lineal de la escala logarítmica

- Alumnos que se confunden al evaluar el valor de las líneas de división secundarias (las cuales son múltiplos enteros del 2 al 9 de la línea de división principal anterior). Esta característica en los diagramas logarítmicos implica que para cada década, existen 8 líneas de división secundarias; mientras que en una escala lineal existen 9 líneas de división secundarias. 


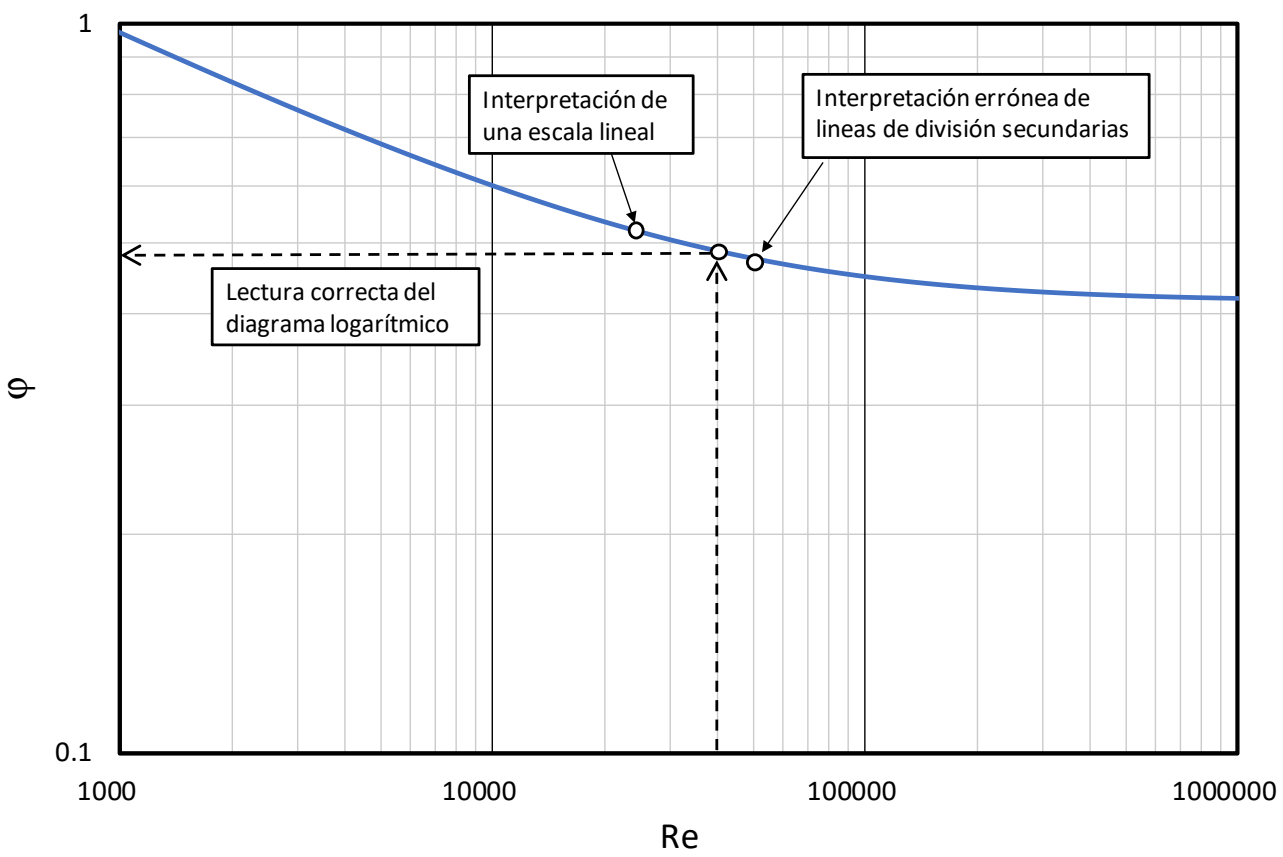

Fig. 2 Ampliación de la zona de respuesta del diagrama logaritmico en el cual se indican las zonas correspondientes a las tres tipos de respuestas proporcionados por los alumnos.

El número de alumnos clasificados en cada uno de estos grupos se indica en la Tabla 1.

Tabla 1. Resultados del análisis factorial

\section{Clasificación de la respuesta}

Alumnos que leen correctamente en el diagrama logarítmico

Alumnos que realizan una interpretación lineal de la escala entre las líneas principales de división

Alumnos que se equivocan al asignar el valor correspondiente a las líneas de división secundarias

Alumnos que no calculan el número Re correctamente (habilidad de lectura no evaluable)

\section{Número de alumnos}

28

11

16

9

Total

En la Figura 3 se representan los porcentajes correspondientes a cada uno de los tres grupos incluidos en el análisis. Si se excluyen los alumnos que no calculan correctamente el valor de entrada al gráfico del análisis realizado en la presente investigación, se infiere que más de un 50\% interpretan correctamente las escalas logarítmicas. Si bien este porcentaje es significativo, cabe enfatizar que los alumnos evaluados se encuentran en tercer curso de grado. Por lo tanto, aquellos estudiantes con errores conceptuales importantes cuentan con pocas oportunidades dentro del grado para su corrección. Entre dichos estudiantes, un $29 \%$ comete errores asociados a un conteo del número de líneas divisorias secundarias 
equivocado. El 20\% restante, realiza una interpretación lineal de la escala, obviando la utilidad de las líneas de división secundarias.

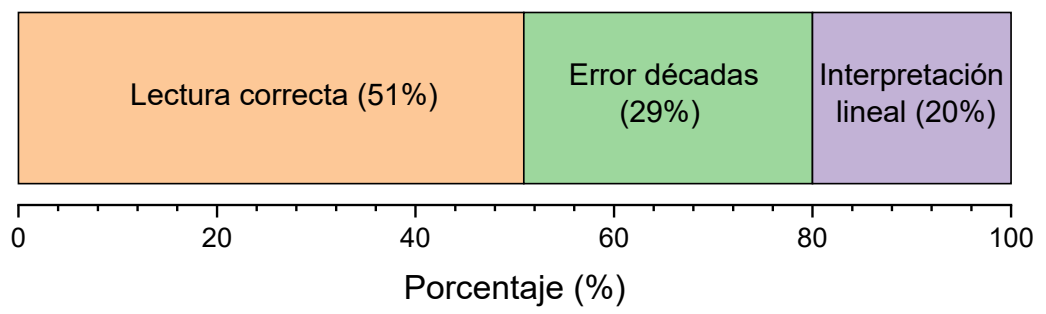

Fig. 3 Diagrama de porcentajes acumulados correspondientes a los tres tipos de respuesta proporcionados por los alumnos que calcularon correctamente el número de entrada a gráfico.

\subsection{Desarrollo de la herramienta PUNTGRAF}

La herramienta de lectura de diagramas PUNTGRAF está pensada para trabajar con diagramas escaneados o creados en formato de gráfico (.jpeg, .png, etc.) realizando transformaciones geométricas y determinados cálculos con las variables transformadas de manera automatizada. En el menú inicial de la aplicación se indican los pasos a seguir para su utilización, tal como se muestra en la Figura 4 (a). A continuación, es necesario indicar el tipo de gráfico con el que se va a trabajar con el programa. Como se puede observar en la Figura 4 (b), existe la posibilidad de escoger entre distintas tipologías de gráfico. Es posible seleccionar un gráfico con ambas escalas lineales, con ambas escalas logarítmicas, un diagrama semilogarítmico o también diagramas triangulares rectángulares y equiláteros.

a)

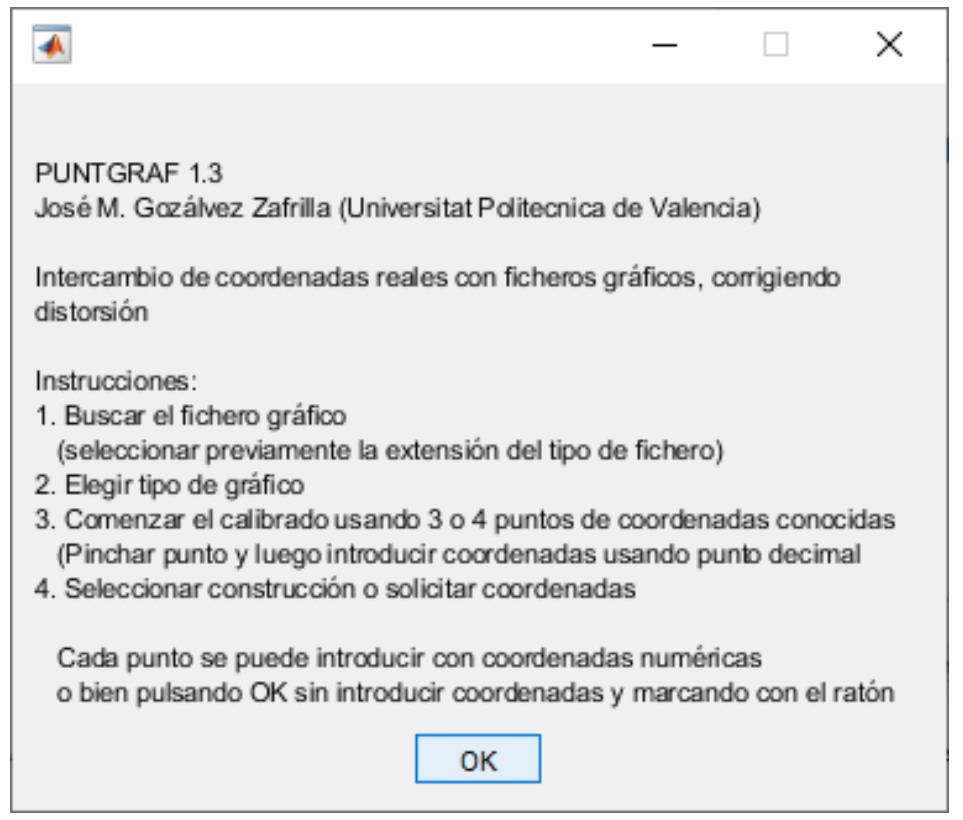

b)

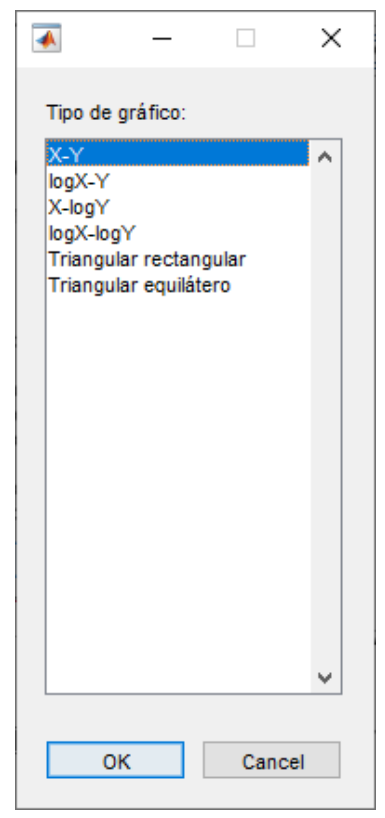

Fig. 4 (a) Pantalla inicial indicativa de los pasos a seguir para la utilización de PUNTGRAF. (b) Selección del tipo de gráfico. 
Una vez seleccionada y cargada la imagen del gráfico es necesario indicar los valores de al menos tres coordenadas conocidas. A modo de ejemplo, la Figura 5 muestra el diagrama proporcionado a los alumnos durante el examen, cargado en el programa PUNTGRAF. El programa realiza un calibrado del gráfico, el cual permite corregir las posibles distorsiones que pueda tener, lo cual ocurre con bastante frecuencia en el caso de gráficos escaneados. En el ejemplo mostrado en la Figura 5 aparece el cuadro de diálogo en el cual se solicita pinchar en el vértice inferior izquierdo del gráfico y proporcionar su valor (1; 0.1). Tras indicar la primera coordenada de referencia, se seguiría calibrando el gráfico indicando, de forma análoga, 2 o 3 vértices adicionales.

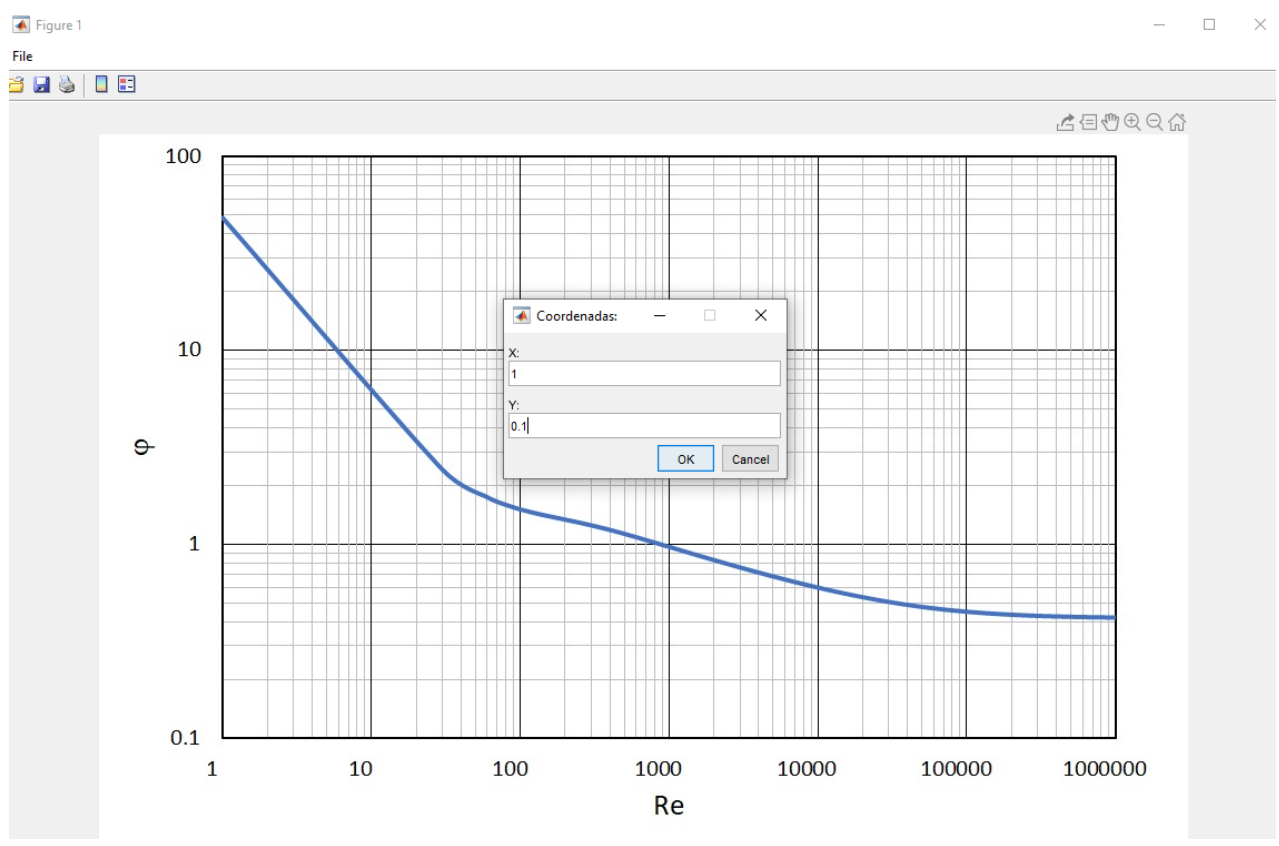

Fig. 5 Calibración del diagrama logarítmico utilizado con los alumnos de tercer curso de Ingeniería Química: indicación de la coordenada correspondiente a la esquina izquierda inferior del gráfico.

Una vez calibrado el gráfico, es posible utilizar distintas funciones del programa. Entre otras, se pueden obtener las coordenadas de un punto, calcular la longitud del segmento que une dos puntos o trazar líneas horizontales y verticales (Figura 6(a)). A modo de ejemplo, se muestra en la Figura 6(b) el procedimiento a realizar para determinar las coordenadas de un punto. Para ello, se pincha con el puntero en el lugar del gráfico del cual se quieren determinar las coordenadas, y el programa devuelve inmediatamente los valores de la abcisa y la ordenada de dicho punto. En el ejemplo mostrado se le ha preguntado al programa por el punto solicitado a los alumnos, pudiéndose comprobar que el valor proporcionado por el programa coincide con el valor correcto $\varphi \approx 0.49$ solicitado a los alumnos. La herramienta PUNTGRAF se ha puesto a disposición de los alumnos en la plataforma online PoliformaT. El programa además de la lectura permite como se aprecia en la Figura 6(a) la realización de determinadas operaciones y construcciones gráficas utilizadas usualmente en ingeniería química. 
a)

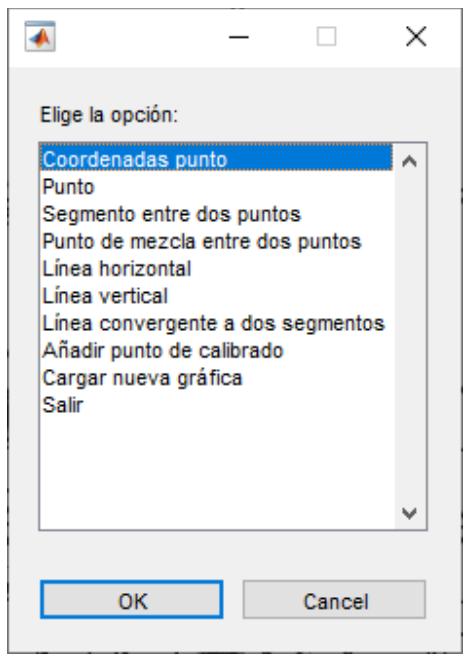

b)

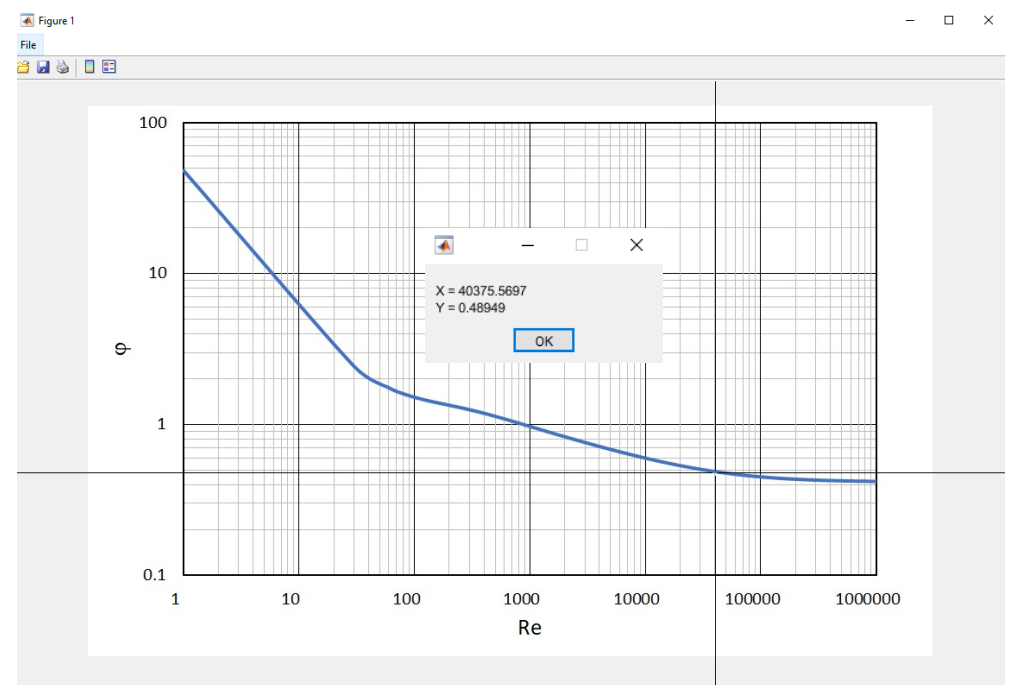

Fig. 6 (a) Menú de selección de la función a utilizar con PUNTGRAF. (b) Obtención de las coordenadas.

Hay que resaltar que el programa no pretende suplir la lectura desde los gráficos, pues el alumno debe saber interpretarlos en cualquier circunstancia.

Las aproximaciones docentes más adecuadas serían:

- Proporcionar el programa previamente a las prácticas y pedirles que lean en los gráficos varios puntos y que utilicen el programa para autocorregirse (formación preliminar).

- Solicitar en primer lugar la lectura directa durante las prácticas y, a continuación, la utilización del programa para autocorregirse (formación directa)

- Utilización exclusiva del programa en prácticas en las que se deban realizar tomas de muchos puntos o en las que el programa apoye determinadas construcciones gráficas. Esta opción sólo se utilizaría en alumnos que muestren ya dominio de la lectura directa de gráficos (uso como herramienta de trabajo)

\section{Conclusiones}

Los resultados obtenidos referentes al análisis de la capacidad de lectura e interpretación de gráficos logarítmicos por parte de estudiantes de tercer curso del grado de ingeniería química nos permiten concluir que:

- Un elevado porcentaje de estudiantes (49\%) realiza una interpretación errónea de los gráficos logarítmicos, siendo por tanto necesario un refuerzo del aprendizaje.

- Los dos tipos de errores cometidos en la lectura de diagramas logarítmicos están relacionados con la tendencia a interpretar los gráficos en escala lineal:

- Una parte importante de dichos alumnos interpreta el espacio entre líneas de división principales de forma proporcional, confundiendo la escala logarítmica con la escala lineal. 
- Otra parte de los alumnos que cometen errores identifican inicialmente de forma correcta que el gráfico está representado en escala logarítmica. No obstante, cometen el error de contar las líneas de división secundarias como si fueran 9 en lugar de 8.

Con respecto al desarrollo de la aplicación PUNTGRAF y su potencial uso para mejorar la precisión de los alumnos en la lectura de diagramas ingenieriles se pueden extraer las siguientes conclusiones:

- El programa PUNTGRAF permite la lectura correcta de distintos tipos de diagramas ingenieriles, incluyendo los diagramas logarítmicos y semilogarítmicos. Además, permite corregir la distorsión que se haya podido producir durante el escaneo del gráfico.

- Se espera que la utilización de PUNTGRAF en próximos cursos permita a los alumnos corregir sus errores de forma autónoma, mejorando el porcentaje de alumnos que son capaces de interpretar correctamente una escala logarítmica y de obtener una lectura precisa de los valores a partir de dichos diagramas.

- PUNTGRAF es una herramienta aplicable tanto para el aprendizaje inicial en los primeros cursos universitarios, como para la corrección de errores en cursos más adelantados (a modo de corrección autónoma de errores).

- El potencial de PUNTGRAF no se limita solamente a la lectura de diagramas logarítmicos, sino que es aplicable a la lectura de otro tipo de diagramas como los triangulares rectangulares y equiláteros, así como a la realización de construcciones y operaciones con gráficos usuales en ingeniería química. La aplicación de dicha herramienta con otros tipos de diagramas ingenieriles está planificada para los próximos cursos.

\section{Referencias}

DePierro, E., Garafalo, F., \& Toomey, R. (2008). Helping students make sense of logarithms and logarithmic relationships. Journal of Chemical Education, 85(9), 1226-1228. https://doi.org/10.1021/ed085p1226

Di Capua, R., Offi, F., \& Fontana, F. (2014). Check the Lambert-Beer-Bouguer law: A simple trick to boost the confidence of students toward both exponential laws and the discrete approach to experimental physics. European Journal of Physics, 35(4). https://doi.org/10.1088/0143-0807/35/4/045025

Dreyfus, T., \& Eisenberg, T. (1990). On difficulties with diagrams: Theoretical issues. In G. Booker, P. Cobb, \& T. N. DeMendicuti (Eds.), Proceedings of the 14th PME conference (pp. 27-34). Mexico Citi.

Furukawa, H., Kato, Y., Inoue, Y., Kato, T., Tada, Y., \& Hashimoto, S. (2012). Correlation of power consumption for several kinds of mixing impellers. International Journal of Chemical Engineering, 2012. https://doi.org/10.1155/2012/106496

Glazer, N. (2011). Challenges with graph interpretation: A review of the literature. Studies in Science Education, 47(2), 183-210. https://doi.org/10.1080/03057267.2011.605307

Heckler, A. F., Mikula, B., \& Rosenblatt, R. (2013). Student accuracy in reading logarithmic plots : the problem and how to fix it. 1066-1071.

Park, E. J., \& Choi, K. (2013). ANALYSIS OF STUDENT UNDERSTANDING OF SCIENCE CONCEPTS INCLUDING MATHEMATICAL REPRESENTATIONS: pH VALUES AND THE RELATIVE DIFFERENCES $\mathrm{OF} \mathrm{pH}$ VALUES. International Journal of Science and Mathematics Education, 11(3), 683-706. https://doi.org/10.1007/s10763-012-9359-7 